\title{
Can stochastic consumer phase models in QMRA be simplified to a single factor?
}

Neves, Maria Ines; Mungai, Sylvester N.; Nauta, Maarten J.

Published in:

Microbial Risk Analysis

Link to article, DOI:

10.1016/j.mran.2017.09.001

Publication date:

2018

Document Version

Peer reviewed version

Link back to DTU Orbit

Citation (APA):

Neves, M. I., Mungai, S. N., \& Nauta, M. J. (2018). Can stochastic consumer phase models in QMRA be simplified to a single factor? Microbial Risk Analysis, 8, 53-60. https://doi.org/10.1016/j.mran.2017.09.001

\section{General rights}

Copyright and moral rights for the publications made accessible in the public portal are retained by the authors and/or other copyright owners and it is a condition of accessing publications that users recognise and abide by the legal requirements associated with these rights.

- Users may download and print one copy of any publication from the public portal for the purpose of private study or research.

- You may not further distribute the material or use it for any profit-making activity or commercial gain

- You may freely distribute the URL identifying the publication in the public portal

If you believe that this document breaches copyright please contact us providing details, and we will remove access to the work immediately and investigate your claim 
Can stochastic consumer phase models in QMRA be simplified to a single factor?

Maria Inês Neves ${ }^{1}$, Sylvester N. Mungai ${ }^{2}$, Maarten J. Nauta ${ }^{1^{*}}$

${ }^{1}$ National Food Institute, Technical University of Denmark, Kemitorvet, Building 202, 2860 KGs. Lyngby, Denmark.

${ }^{2}$ Kenya Bureau of Standards, P.O. Box 2138, Nakuru 20100, Kenya.

${ }^{*}$ corresponding author

Keywords: food chain risk assessment, relative risk, consumer, model

\section{Highlights:}

- Stochastic consumer phase models are compared with simplified surrogate models.

- Case studies for consumer phase models for Campylobacter, Salmonella and Listeria are analysed.

- The relative risk estimates after intervention in the food chain are higher for stochastic models.

- Neglecting the variability between consumers may overestimate the predicted effect of intervention measures. 


\section{Abstract}

In quantitative microbiological risk assessment (QMRA), the consumer phase covers the part of the food chain following production and retail, where the consumer transports, stores, prepares and consumes the food products considered. These consumer practices have a crucial impact on exposure, and a consumer phase model (CPM) needs to be included in a QMRA to allow an evaluation of the effectiveness of intervention measures in food production and processing in terms of human health risk. However, the development of a CPM is complex because consumer practices can be highly variable and data are scarce. So far, it is unclear to which extent CPMs need to include data on variability and detailed descriptions of the stochastic processes that may result in exposure. We therefore compared the performance of published stochastic CPMs with a simple surrogate CPM that assumes a proportional linear relation between concentration at retail and ingested dose, described by a constant factor.

A comparative study was performed for different pathogens and different food products: Campylobacter in broiler meat, Salmonella in minced pork and pork cuts and Listeria in smoked salmon. Published stochastic CPMs were re-implemented and their equivalent surrogate models were derived, basing the value of the constant surrogate model factor on the absolute risk estimate from the stochastic model. The performances of the models were evaluated by comparing the effects of hypothetical intervention measures that reduce the mean or the standard deviation of the distribution of concentrations at retail. These effects were expressed in terms of relative risk estimates, as estimated in the risk assessments using the simplified and the stochastic CPMs.

Results showed that after interventions that result in a reduction of the mean or standard deviation of the distribution of concentrations at retail, the relative risk estimates obtained for the simple surrogate models are always lower than those of the stochastic CPMs, which means that simplified models tend to overestimate the effects of interventions. The difference was largest in the Listeria model, where growth during storage is expected to be the dominant process. It was found that for interventions affecting the prevalence only, a simplified surrogate CPM performs similarly to a stochastic CPM.

We concluded that the use of a simple surrogate CPM, which does not include the variability inherent to consumer practices, may lead to an overestimation of the effect 
of intervention measures in a QMRA, especially in these interventions affect the concentrations. For adequate risk assessment, it may therefore be necessary to include the variation in consumer practices (e.g. variation in storage time and temperature, cooking time and temperature and cross-contamination), as described in more realistic and more complex CPMs, definitely if this variation is expected to be large. 


\section{Introduction}

In quantitative microbiological risk assessment (QMRA), the consumer phase is the part of the food chain following the production and retail, when the consumer transports, stores, prepares and consumes produced food (Nauta \& Christensen, 2011). This step is different from all the other steps of the food chain, because it is associated with high variability in human behaviour and because consumer food safety practices cannot be enforced by legislation (Nauta et al., 2009). Next, representative quantitative data on consumer food handling practices are scarce. This is due to the difficulty of obtaining unbiased, representative data on human behaviour in the domestic setting in general (Redmond \& Griffith, 2003), which restrains research in the consumer phase. Also, there is a high variability in food handling practices, and cultural and social differences between the considered population groups in relation to food preparation practices need to be accounted for (Nauta et al., 2009). Despite these challenges, a consumer phase model (CPM) always needs to be included in a risk assessment: to allow an evaluation of the effectiveness of intervention measures in food production and processing in terms of human health risk, the step from contaminated food product to exposure has to be taken and always includes a consumer that handles the food product. In some cases, food handling may not impact the bacterial concentration in food and the consequential exposure, but most often it does.

To this day, several CPMs have been developed. These may differ substantially in terms of complexity, depending on the purpose of the QMRA and the availability of data. Some may only include a few simplifying assumptions, while others may describe in detail the food handling practices, their frequencies of occurrence and use many data sources (Zwietering, 2009).

An important question to answer in the development of CPMs is to what extent these models need to include a detailed description of the processes that may result in exposure (Nauta and Christensen, 2011). The study from Nauta et al. (2009) suggests that "there is no alternative but for a probabilistic approach to risk assessment models of the consumer phase". This would imply that one should always use a stochastic CPM to obtain accurate risk estimates in QMRA. Yet, for practical reasons, the use of a simplified CPM may be preferable, so it would be important to know to what extend such surrogate models perform sufficiently for a reliable QMRA. 
An example of a surrogate model is the one used by Duarte et al (2016) in a study about the effect of carcass decontamination on the risk for consumers, later applied by Bollerslev et al. (2017) as well. In their model, it is assumed that the survival of Salmonella in pork meat, from the carcass to the consumed serving, is identical for all servings. A constant factor a summarises the reduction in concentration from carcass to ingested dose due to transfer, growth, cross contamination and survival of Salmonella in pork meat. The value for the $a$ factor is obtained by linking the distribution of concentrations found on the raw meat with a dose-response relation and an epidemiological estimate of the incidence of salmonellosis attributable to pork. By using this surrogate, it is not necessary to make additional assumptions on the survival of the pathogen. However, it is stated in the study that the assumption of an identical effect of consumer food handling for all serving is unlikely to be correct (Duarte et al., 2016). This is based on the fact that for example studies for Campylobacter on broiler meat have shown that there is variation present in transfer and survival during the consumer phase, and that it is essential to consider this in a risk assessment (Duarte et al., 2016; Nauta and Christensen, 2011; Nauta et al., 2009). However, the performance of this surrogate model in comparison to a stochastic CPM has never been evaluated.

The objective of this study is therefore to evaluate the performance of a simple surrogate model like the "a-factor" model (Duarte et al. 2016) by comparing it with previously published stochastic consumer phase models for different pathogens and different food products. As in Nauta and Christensen (2011), this evaluation is done by comparing relative risk estimates for the effects of hypothetical interventions that modify the distribution of concentration at retail (i.e. the last stage before the consumer phase). In this approach, similar relative risk estimates suggest that the performance of surrogate models is adequate, whereas dissimilar estimates show that simplification of the CPM may yield misleading conclusions in QMRA.

\section{Methods}

\subsection{Generic Modelling approach}

In QMRA, the mean risk per serving is the mean probability of illness from all servings 
$\operatorname{Risk}_{D}=\sum_{D=0}^{\infty} Q_{i l l}(D) f(D)$

where $D$ is the (discrete) dose, $f(D)$ is the probability distribution of doses obtained from the exposure assessment (including uncontaminated servings, i.e. $D=0$ ) and $Q_{\text {ill }}(D)$ is the probability of illness from exposure to dose $D$, i.e. the dose-response relation. Usually, the dose D on a serving cannot be measured and is obtained from a consumer phase model (CPM) where D is obtained from a concentration (C) higher up in the food chain (e.g. at retail). If $C P M(C)$ represents the function that relates $D$ to $C$ :

$\mathrm{D}=\mathrm{CPM}(\mathrm{C})$

and consequently the mean risk

Risk $_{C}=\int_{0}^{\infty} Q_{i l l}(C P M(C)) g(C) d C$

Here, $g(C)$ represents the probability density function of the concentrations $C$ (a real number). Note that the subscript for Risk only indicates how the risk is calculated, the value obtained for eq. (1) and eq. (3) should be the same (Risk ${ }_{D}=$ Risk $_{C}$ ). In this paper, we explore the impact of simplifying the consumer phase model as in Duarte et al. (2016), by using

$D=a C$

instead of eq. (2), where $a$ is the "a-factor", a surrogate for the CPM.

Hence,

$\operatorname{Risk}_{a}=\int_{0}^{\infty} Q_{i l l}(a C) g(C) d C$

To obtain an estimate for $a$ for different CPMs and different dose-response relations $\left(Q_{i l l}(D)\right)$, we first calculate Risk and then set Risk ${ }_{c}=$ Risk $_{a}$ 
$\operatorname{Risk}_{C}=\int_{0}^{\infty} Q_{i l l}(a C) g(C) d C$

where $a$ is the only unknown and can be obtained numerically, for example by approximating the integral and using the Excel Solver add-in, as in Duarte et al. (2016).

To compare the performance of the CPMs and the surrogate models, we study the effect of hypothetical changes in the distribution of concentrations $\mathrm{C}$. These changes can represent the effect of interventions implemented to reduce the risk. If the alternative risk estimate obtained by using an alternative distribution of concentrations is Risk ${ }^{*}$, we

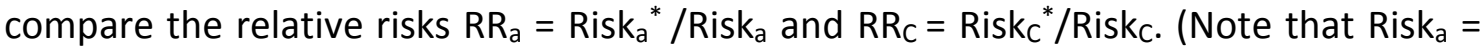
Riskc after using the procedure described above, eq. (6), however Riska (eq. (5)) may be different from Riskc ${ }^{*}$ (eq. (3)) ). Such relative risks are frequently studied as an important outcome of QMRAs, and have the advantage that they are less uncertain than absolute risk estimates, as uncertainties are partially cancelled out. If the relative risks $R_{a}$ and $R R_{c}$ are similar, the surrogate can be considered an appropriate alternative to the CPM, otherwise it can not.

Four intervention scenarios are compared, where the control measures are assumed to be implemented somewhere along the food production chain, at primary production or during industrial processing. Therefore, they do not affect the CPM itself (Nauta \& Christensen, 2011).

- Scenario 1: 0.5 log reduction in the mean of the concentration at retail.

- Scenario 2: 1 log reduction in the mean of the concentration at retail.

- Scenario 3: 0.5 decrease in the standard deviation of the concentration at retail.

- Scenario 4: 0.5 increase in the standard deviation of the concentration at retail. Scenarios 1 and 2 represent practical control measures that are believed to affect the mean concentrations of the pathogen, like for example, decontamination of broiler meat during industrial processing to reduce Campylobacter (Gellynck et al., 2008; Havelaar et al., 2007). Scenario 3 simulates, for example, implementation of protocols where heavily contaminated meat products are diverted from the fresh broiler meat production chain to reduce Campylobacter, which results in a decrease of the standard 
deviation (Nauta et al., 2009; Nauta \& Havelaar, 2008). Scenario 4 could, for example, represent a situation of protocols where less control samples are taken, which results in more variation in the concentration of the pathogen in food, and increases the standard deviation.

\subsection{Selection of case studies}

Five case studies were performed, on the basis of CPMs and dose response models found in the literature, covering three well known foodborne pathogens with different characteristics in the consumer phase: typically, during transport and storage, Campylobacter does not grow, Salmonella has a growth potential with temperature abuse, and Listeria can even grow at low temperatures. For all pathogens cross contamination and inactivation may occur, but for Listeria the selected CPM only included growth during storage.

The first case study applied the Campylobacter in broiler chicken CPM published by (Nauta et al., 2008). This study considered bacterial transfer from inoculated chicken breast fillets to salads in ready-to-eat chicken salad prepared at home by consumer volunteers, and relied on other studies (De Jong et al., 2008; Van Asselt et al., 2008) that used Lactobaccili as a tracer for Campylobacter. This CPM performed as a representative CPM in the analysis of Nauta and Christensen (2011) and it has been used in other studies as well (EFSA 2010, Nauta et al 2012, Nauta et al 2015).

For Salmonella in pork, we used two CPMs based on Swart et al (2016). These CPMs are part of a full risk assessment model for Salmonella in the pork production chain in selected European Union member states (Hill et al., 2011; Swart et al., 2016; Snary et al., 2016). In this model three types of pork meat are considered: minced pork, pork cuts and fermented sausages. Here we use the CPMs for minced meat and pork cuts, which allows us to compare the effect of using different meat products derived from the same raw material. Fermented sausages are not included as their risk mainly originates from failed fermentation, which falls outside the consumer phase.

For Listeria monocytogenes, we used a CPM based on the exposure assessment of a study performed by Berjia et al. (2013), a risk-benefit assessment of cold-smoked salmon (CSS). In this study, the risk of Listeria monocytogenes is evaluated against the 
benefits of the intake of omega- 3 fatty acids in Denmark. The model described is deterministic, and was made stochastic by using a growth model described by Pouillot \& Lubran (2011). Two different dose-response models are used, one for the healthy population and one for the sensitive population, therefore for Listeria two different case studies can be compared as well.

\subsection{Case studies}

For each case study, we describe (1) a distribution of the concentrations at retail, $g\left(C_{\text {ret }}\right)$, including the prevalence, as input for the CPM; (2) the stochastic CPM, CPM( $\left.\mathrm{C}_{\text {ret }}\right)$; and the dose response relation $Q_{\text {ill }}(D)$.

To get a risk estimate, Riskc (eq. (3)) is estimated by Monte Carlo simulation, using the Excel add in @Risk, version 6.1 (Palisade). 100,000 samples are taken from $g\left(C_{\text {ret }}\right)$, given that the product at retail is contaminated as indicated by the prevalence, and used as input in the stochastic CPMs. The doses obtained from the CPM (one per sampled $\mathrm{C}_{\text {ret }}$ ) are fed into the dose-response relation $Q_{\text {ill }}(D)$. The mean of all resulting probabilities of illness are multiplied with the prevalence, which provides the mean risk of illness.

Next, by solving eq. (6), values for $a$ are obtained for each case study, to use in the surrogate models. Relative risks are calculated for the four intervention scenarios, for both the stochastic CPMs and the surrogate models before and after intervention, using 1,000,000 iterations in @Risk.

\subsubsection{Campylobacter}

As in Nauta and Christensen (2011), with a probability equal to prevalence $p_{\text {prev }}=0.25$, the 10-based log of the concentration at retail $\log C_{\text {ret }}$ is sampled from a Normal distribution with mean $=1.5 \log \mathrm{cfu} / \mathrm{g}$ and standard deviation $(\mathrm{SD})=1.2$, otherwise $C_{\text {ret }}$ is $0 \mathrm{cfu} / \mathrm{g}$. According to the CPM, the number of Campylobacter (cfu) on one portion of consumed meat, $N_{\text {portion, }}$ is defined by a Poisson distribution

$N_{\text {portion }} \sim$ Poisson $\left(C_{\text {ret }} \times W_{c}\right)$ 
where $\sim$ represents "is a sample from," and portion sizes, $W_{c}$, are sampled from a lognormal distribution with mean $=189 \mathrm{~g}, \mathrm{SD}=127$, and maximum portion size of $1 \mathrm{~kg}$. (Christensen et al. 2001).

In an observational study, Nauta et al (2008) obtained a data set describing the variability of transfer rates from raw meat to salad and an empirical distribution of 55 transfer rates $p_{t r}$ was provided (described in Nauta \& Christensen (2011)). This yields a distribution of doses by applying

$D \sim \operatorname{Binomial}\left[N_{\text {portion }}, p_{t r}\right]$

A Beta-Poisson dose-response model from FAO/WHO (2009) is used to describe the probability of infection from an ingested dose $D$. As the classic Beta-Poisson model describes the response in terms of probability of infection, a standard multiplier of $\mathrm{P}_{\mathrm{ill}} \mathrm{inf}$ is used to calculate the probability of illness $Q_{\text {ill., }}$ based in studies from Black et al. (1988) and from FAO/WHO (2009).

$Q_{i l l}(D)=P_{\text {ill } \mid \text { inf }} \times\left(1-\left(1+\frac{D}{\beta}\right)^{-\alpha}\right)$

Where, based in studies from Black et al. (1988), FAO/WHO (2009), $\alpha=0.145$ and $\beta=$ 7.59 and $\mathrm{P}_{\mathrm{ill} \mid \mathrm{inf}}=0.33$.

\subsubsection{Salmonella}

The inputs applied to Salmonella CPM were obtained from Bollaerts et al. (2009): with a probability equal to prevalence $p_{\text {prev }}=0.12$, the 10 -based log of the concentration at retail, $\log C_{\text {ret, }}$ is sampled from a Normal distribution with mean= $1.4 \log \mathrm{cfu} / \mathrm{g}$ and $\mathrm{SD}=$ 0.7, otherwise $C_{\text {ret }}$ is $0 \mathrm{cfu} / \mathrm{g}$. As in Swart et al. (2016), portion sizes $\mathrm{W}_{\mathrm{s}}$ are fixed at $146 \mathrm{~g}$ for pork cuts and $125 \mathrm{~g}$ for minced meat, so the initial number of cfu per portion $\mathrm{N}_{0}=$ $\mathrm{C}_{\text {ret }} \times \mathrm{W}_{\mathrm{s}}$. Both CPMs, for pork cuts and minced meat, as described by Swart et al 2016, consider three processes: transport and storage, preparation and heating, and are 
presented in Figure 1. The transport and storage process is modelled identically for both products. Swart et al. (2016) use Baranyi's dynamic growth model. As it is difficult to apply this specific model in @ Risk and the specific choice of the growth model is not of great importance for our purposes, we apply an exponential growth model as a primary growth model and a square root model for the temperature (Ratkowsky 1982) as a secondary growth model in this study:

$\log N_{s t}=\log N_{0}+\left(b\left(T_{t r}-T_{\min }\right)\right)^{2} t_{t r}+\left(b\left(T_{s t}-T_{\min }\right)\right)^{2} t_{s t}$

where $T_{t r}, T_{s t}, t_{t r}$ and $t_{s t}$ are the randomly sampled temperature $(T)$ and time (t) during transport (tr) and storage (st), using the same distributions as Swart et al. (2016). $\mathrm{N}_{\text {st }}$ is the number of cfu per portion after transport and storage. The constant $b$ and minimum growth temperature $T_{\min }$ are parameters obtained from the data on measured growth rates at four different temperatures, presented by Swart et al. 2016 (see their table 1). Values $\mathrm{T}_{\min }=4.00^{\circ} \mathrm{C}$ for pork chops and $\mathrm{T}_{\min }=2.48^{\circ} \mathrm{C}$ for minced meat were obtained by extrapolation of the regression line between the measured growth temperatures and the associated square roots of the growth rate (Swart et al 2016); values $b=0.0360$ for pork chops and $b=0.0261$ for minced meat were obtained by taking the average of the four estimates of $b$ obtained from the four observations.

Preparation and heating are modelled differently in both Salmonella CPMs:

\section{Pork cut model}

The preparation of pork cuts considers that the product is prepared in a domestic environment. A ready-to-eat salad may be prepared as a side dish along with the pork cut. Transfer of Salmonella can occur via a cutting board, knife and hands that are not washed, and via the water tap. The likelihood of washing is defined, and transfer coefficients are used that describe the fraction of Salmonella that migrates from one object to another. Survival rates describe the effectivity of the washing steps.

The transfer matrices containing transfer rates between knife, board, hands and tap , are described in detail by Swart et al. (2016).More specific references are given in Figure 1. Inadequate heating (i.e. heating that does not inactivate all Salmonella bacteria) is 
not considered for pork cuts, as it is assumed that Salmonella is completely inactivated since it is only present on the outside of the product (step 5 in Figure 1). For this reason, it is assumed that only Salmonella present in the side dish are ingested.

\section{Minced meat model}

The preparation of minced meat is divided in two steps: the meat is handled to prepare hamburger patties or meat balls and afterwards a salad is prepared. As in Swart et al. (2016), the cross-contamination process for minced meat was a simplified version of the pork cuts model, since in this model a knife is not used, the survival rates on board and hands were set to zero, and the tap was not included as a transfer route. Details on the final model are given by Swart et al (2016).

For heating, the cooking model described by Bergsma et al. (2007) is used, which includes the following steps (Figure 1, step 5):

- $\quad$ Cooking of one side of the patty for one minute at high temperature.

- The product is turned, and it is assumed that a crust has formed, creating a perfectly insulating patty.

- $\quad$ The product is cooked for a few more minutes at a lower temperature.

The model used describes a polynomial fit of the data (Swart et al 2016)

$\log \left(\mathrm{N}_{\text {mcook }}\right)=\log \left(\mathrm{N}_{\mathrm{mcc}}\right)-4.36 \times 10^{-3} t^{3}-1.02 \times 10^{-2} t^{2}+4.73 \times 10^{-2} t$

Where $\mathrm{N}_{\mathrm{mcc}}$ is the number of Salmonella per patty after handling the product with transfer of some cells to the hands and the cutting board, $t$ is the cooking time, and $\mathrm{N}_{\text {mcook }}$ is the number of cfu per patty after cooking. For each CPM (pork cut and minced meat), the dose $D$ is obtained as indicated in step 7 of Figure 1.

The Beta-Poisson dose-response model from FAO/WHO (2002) is used to describe the probability of infection from an ingested dose $D$, i.e. the same model as in eq. (9), with $\alpha=0.1324$ and $\beta=51.45$. As the parameters of the dose-response model were obtained 
from outbreak data, the results can be interpreted to describe the probability of illness from a certain dose so $\mathrm{P}_{\mathrm{ill}}$ inf $=1$.

\subsubsection{Listeria}

In the study of Berjia et al (2012), the initial concentrations of L. monocytogenes in cold smoked salmon are $\{0.5,1.5,2.5\} \log \mathrm{cfu} / \mathrm{g}$ with prevalences $\{0.28,0.05,0.01\}$ (Jørgensen and Huss 1998). These initial concentrations $C_{0}$ were modified to better describe the variability in the model and are now defined by a Normal distribution with mean=1.2189 log cfu/g and SD=0.8. Here, the mean was calculated by adding the products of the indicated initial concentrations and their respective prevalences, (Jørgensen and Huss 1998). The value of standard deviation (SD) $=0.8 \log _{10}$ units was chosen based on ILSI (2010), where it is suggested to use this value as a default value to describe the standard deviation of a batch, when no better data or more specific information on a batch is available.

In the CPM, the portion size has a fixed value of $W_{L}=23 \mathrm{~g}$. Next, growth during storage is modelled by applying a three-phase linear primary growth model (Buchanan et al., 1997), model \# 4 published by Pouillot \& Lubran (2011) :

$C_{\text {end }}=\min \left(C_{0}+\frac{\mu}{\ln (10)} \times \max (t-\lambda, 0), M P D\right)$

where $C_{0}\left(\log _{10} \mathrm{cfu} / \mathrm{g}\right)$ is the initial concentration of L. monocytogenes in the CSS at the beginning of the storage; $\mu$ is the specific growth rate of L. monocytogenes per day $\left(d^{-1}\right)$; $t$ is the duration of the storage (d); $\lambda$ is the lag time (d); MPD is the maximum population density $\left(\log _{10} \mathrm{cfu} / \mathrm{g}\right)$. As in (FDA/FSIS, 2003), model \#4 used $\lambda=0$ days and MPD $=7.27$ (Delignette-Muller et al., 2006). As a secondary model for $\mu$ a square root model (Ratkowsky et al., 1982) was used:

$\mu=\mu_{\text {ref }} \times\left(\frac{T-T_{\min }}{T_{r e f}-T_{\min }}\right)^{2}$ 
with $\mu_{\text {ref }}=6.19 \mathrm{~d}^{-1}$ (FDA/FSIS, 2003) for $\mathrm{T}_{\text {ref }}=25^{\circ} \mathrm{C}$ (Delignette-Muller et al., 2006) and a constant $\mathrm{T}_{\min }=-1.18^{\circ} \mathrm{C}$ (FDA/FSIS, 2003).

The storage temperature (T) was obtained from FAO/WHO (2004; Table A5.3). The storage time $t$ is described by a Normal distribution with mean=14 days (Berjia, 2013) and SD = 3.5 (assumption)

In this case, the expected dose is obtained by

$\mathrm{D}=\mathrm{W}_{\mathrm{L}}{ }^{*} \mathrm{C}_{\text {end }}$

An exponential dose-response model is used to estimate the probability of infection by L. monocytogenes (FAO/WHO 2004):

$P_{i l l}=1-e^{-r D}$

where, $P_{\text {ill }}$ is the probability of severe illness, $D$ is the dose, and $r$ is the parameter that defines the dose-response relation for the population being considered. As in (FAO/WHO 2004) and Ross et al. (2009), for the healthy population $r=$ $2.37 \times 10^{-14}$, for the susceptible population $r=1.06 \times 10^{-12}$.

\section{Results}

\subsection{Risk estimates and a-factors}

Using the modelling approach as explained in 2.1, values of the a-factor are derived for each of the five case studies. First the mean risk Riskc is estimated by combining the input distribution of concentrations, the CPM and the dose-response relation, and using eq. (3) Then, $a$ is obtained from numerically solving eq. (6). The results are given in Table 1. It shows that the risk estimates for Campylobacter and Salmonella are larger than those for Listeria, but the values for the a-factor are much larger for Listeria. The first is mainly due to the dose-response relations, the second is due to the fact that the Listeria CPM describes growth (an increase in concentration), whereas those for Campylobacter and Salmonella describe inactivation and transfer as dominant processes, which give a 
decrease in concentration.

\subsection{Relative risk estimates}

The relative risk estimates for the stochastic CPM and the surrogate model are given in Figure 2. It shows that the surrogate models always give a lower estimate of the relative risk than the CPMs, if the mean or standard deviation of the distribution at retail is reduced. The difference between the two relative risk estimates is largest in the Listeria models. The relative risks obtained with the Salmonella models for pork cuts and minced pork patties are identical, even though they represent different products. Similarly, the relative risks of the two Listeria models are shown to be the same for the healthy and the susceptible population. For Salmonella and Campylobacter, the difference between the stochastic- and surrogate-CPM relative risks in the interventions with 0.5 and 1 log reduction of the mean initial concentrations are similar, whereas for Listeria this difference increases with increased reduction of the mean. An increased standard deviation (sd) gives larger relative risk values for the surrogate models, which is consistent with a smaller relative risk for a reduction in sd.

\subsection{Stochastic CPMs and surrogate models compared}

To understand the difference between the performance of the stochastic CPM and the surrogate model, they are compared in more detail.

In the stochastic CPMs, the relation between the ingested dose and the concentration at retail is not a constant factor $(a)$, but it varies stochastically between servings, depending on the CPM models used to describe transfer, cross-contamination inactivation and/or growth. So instead of $D=a C_{\text {ret }}$ (eq. (4)), $D=C P M\left(C_{\text {ret }}\right.$ ) (eq.(2)). For the surrogate model $\mathrm{D} / \mathrm{C}_{\text {ret }}=a$ is constant, but for the stochastic model $\mathrm{D} / \mathrm{C}_{\text {ret }}$ will vary between model iterations, i.e. between servings. Figure 3 shows the cumulative distributions of the values of $\log \left(D / C_{\text {ret }}\right)$ for the four indicated CPMs. The a- factor value for each model is given by the black dot. Note that $\log \left(D / C_{\text {ret }}\right)=0$ implies that $D=$ $C_{\text {ret, }} \log \left(D / C_{\text {ret }}\right)>0$ implies $D>C_{\text {ret }}$ and $\log \left(D / C_{\text {ret }}\right)<0$ implies $D<C_{\text {ret. }}$ Here, $D$ is expressed per serving, $C_{\text {retail }}$ is expressed per $\mathrm{g}$. 
Figure 3 reflects that the Listeria CPM is a growth model that gives a variable increase in concentration. In the other CPMs, inactivation and transfer are the predominant factors, and values log $\left(D / C_{\text {ret }}\right)>0$ are only found in cases where the effect of growth and the serving size are dominant $(<10 \%$ of the exposures). In the Salmonella models, cross-contamination can be absent and inactivation can be complete (for PC, not for MM, which explain the difference in shape of the curves); in the Campylobacter model transfer is always present (although in practice the transfer rate may be too low to transfer any cfu). In all models, the a-factor values do not correspond to the median (or mean) of the distribution, but correspond to an upper percentile (67\% for the Campylobacter CPM, > 80\% for the other CPMs).

\section{Discussion}

Consumer phase models (CPMs) are an essential step in Quantitative Microbiological Risk Assessment (QMRA), linking the prevalence and concentrations at retail to the ingested doses. The large variation in consumer food handling practices and scarcity of data to use in a risk assessment imply that several subjective and simplifying assumptions are made when a CPM is constructed. It is unclear to what extent these simplifications are justified. In this study, we therefore compared the performance of some published stochastic CPMs with a highly simplified CPM (the "a-factor" model) that ignores the variability in the effect of food handling, storage and heat treatment by consumers.

If the mean or standard deviation of the distribution of bacterial concentrations at retail is reduced, a comparison of the stochastic CPMs and the surrogate "a-factor" models shows that the surrogate models always give a lower estimate of the relative risk than the stochastic CPMs. This means that the simplified approach, that neglects the variability between consumers, leads to an overestimation of the effect of interventions in the food chain before retail, that reduce the mean and/or standard deviation of concentrations. Hence, a simplified approach may result in an over-optimistic assessment of the effect of risk mitigation strategies.

The finding that variability negatively impacts the expected effect of interventions is in 
agreement with the result of Duarte et al. (2016) who found that inclusion of variation in the effect of decontamination negatively affects its impact in terms of risk reduction. Our results suggest that the use of a stochastic CPM, which includes the effect of variability at the consumer phase, will only strengthen this impact and result in a risk reduction that is lower than the one predicted in that study.

The microbial dose-response models used are linear at low doses (Haas 2002). This implies that, for interventions reducing the mean concentration by 0.5 and 1 log, at low doses one might expect relative risk estimates of 0.316 and 0.1 . Figure 2 shows that these predictions are very close to the values found for the "a-factor" model for Listeria, but all other relative risk estimates are larger. Apparently, in the other models the doses found in the exposure assessment are not low enough to assume linearity and the variability in doses includes too many high doses where the linearity is violated, especially for the stochastic models.

The largest difference between the two relative risk estimates is found in the Listeria models that exclusively describe growth. Listeria is also characterized by a low virulence (a low value of the r-parameter in the dose response relation) and as a consequence the risk estimate will be sensitive to high exposures, which occur more frequently when the variability in doses, expressed on the log-scale, increases. This result indicates that using a simplified surrogate model for Listeria, where the tail of the distribution of exposures is cut down, could have great impact on the assessed effect of interventions.

Another observation made for Listeria is that, in terms of relative risks, the results for the healthy population and the sensitive population are highly similar. This is expected, as the models only differ in the dose response, and this difference is only proportional. For Salmonella, we used different stochastic CPMs for two different pork products: minced meat and pork cuts. For these two products, the prevalence and concentrations at retail and the dose-response relations are identical. The results show that these two CPMs perform very much similar when relative risk estimates after intervention are compared (see Figure 2). This is probably a consequence of the fact that the right hand tails of the distributions shown in Figure 3 are overlapping, i.e. similarly high doses (which have most impact on the risk) occur a similar number of times in both products. This result suggests that, for the relative risk estimate after intervention, the precise definition of the stochastic CPM is less relevant than the question whether variability is 
included in the CPM at all. It may even imply that, for this purpose, it is not necessary to develop specific CPMs for all specific pork products. However, as the risk estimate for the two pork products are almost the same (See table 1), and pork products that are processed differently may yield different risks, this hypothesis demands further research.

In this study, the value of the a-factor is derived from a risk estimate that is obtained from a QMRA that includes a stochastic CPM. This is different from the approach used by Duarte et al. (2016), who use an independent epidemiological risk estimate to derive the value of $a$. The value obtained by them for Salmonella in pork in Denmark is 0.000031 (Bollerslev et al. 2017), which is considerably lower than the values obtained by us ( 0.0798 and 0.0770 , see Table 1$)$. This confirms that QMRA risk estimates are often higher than those derived from epidemiological data (Havelaar et al. 2008). In addition, one might argue that the relationship between the stochastic and deterministic results could change if the a-factor value was obtained from epidemiological data as in Bollerslev et al. (2017). Therefore, Salmonella model was run with a-factor value of 0.000031 and the corresponding relative risks were calculated. The relative risks obtained using this approach were, however, very similar to the ones obtained with afactors calculated by us (data not shown).

Figure 3 illustrates that the a-factor values obtained from numerically solving eq. (6) do not correspond to the median (or mean) values of the distributions of values obtained for $D / C_{\text {ret }}$ describing the effect of the stochastic CPMs, but are located in one of the upper percentiles. One reason for this finding is that there is no analytically solvable relation between $a \mathrm{C}$ and $\mathrm{CPM}(\mathrm{C})$. However, the fact that the numerically obtained value of the a-factor lies in the upper percentiles confirms the notion that the high value tail of the distribution of concentrations is of crucial importance for the mean risk estimate, and therefore confirms that variability should be taken into account in risk assessment. One could argue that the relationship between the stochastic and deterministic results could change if the a-factor value was closer to the mean or median of the distributions in Figure 3. For this reason, Salmonella model was run with a-factor values corresponding to the median of the distributions in Figure 3 and relative risks were calculated. The relative risks obtained were very similar to the ones obtained with previous a-factors, so we can conclude that using the median of the distributions doesn't 
impact the relative risks (data not shown).

In this study, we focus our analysis on interventions targeting the bacterial concentrations on food products, not the prevalence of contaminated products. However, risk mitigation strategies may be specifically directed at reducing the prevalence, for example by reducing the prevalence of contaminated broiler flocks or slaughter animals. For interventions affecting the prevalence at retail only (and not the distribution of concentrations in the contaminated products at retail), the simplified "afactor" CPM is expected to perform equally well as a stochastic CPM. This can be explained as follows:

First, note that $\mathrm{g}(\mathrm{C})$ in eq. (3) and further includes uncontaminated products. If only contaminated products are considered, with distribution of concentrations $\mathrm{g}^{*}(\mathrm{C})$, and the prevalence of these contaminated products is $p$, eq. (3) becomes

$\operatorname{Risk}_{C}=p \int_{0}^{\infty} Q_{i l l}(C P M(C)) g^{*}(C) d C$

Similarly, eq. (5) becomes

$\operatorname{Risk}_{a}=p \int_{0}^{\infty} Q_{i l l}(a C) g^{*}(C) d C$

with the prevalence outside the integral in both equations, the estimated effect of any intervention affecting the prevalence only will have the same proportional effect on the risk estimate of both the stochastic and the surrogate CPM. It is easy to see that this proportionality remains when relative risk estimates are considered.

The main implication of our findings in this study is that a simplified approach towards the CPM, which ignores the variability in effect of consumer food handling, storage and heat treatment, may be insufficient for QMRA. The question that comes up next is to what extent the overestimation of the effect of interventions is relevant for risk management (which is the same question as "when should we consider the difference between the relative risk estimates of the stochastic CPM and the simplified model too large?"). One might argue that, given the uncertainties attending QMRA in general, a relatively small overestimation of this effect does not justify the effort of developing a 
stochastic CPM that undoubtedly needs simplifying assumptions as well. This clearly depends on the specific purpose of the risk assessment, as well as on pathogen and food product considered, as illustrated by the differences observed for the case studies described in this paper.

The case studies were chosen based on existing published stochastic consumer phase models (CPMs). Even though they represent different pathogens and processes, they may not be representative for all potential CPMs, for example because they only involve one or a few processing steps and were built in the context of developed countries, accounting for western handling practices. One could therefore argue that our case studies are too similar and too simple, and that results might change if CPMs built for a wider range of food storage an preparation practices were used instead (eg. different refrigeration or different cooking approaches). Although these different practices may undoubtedly have an important impact on the risk estimates (Riskc), the impact on the relative risk estimate after intervention $\left(R R_{c}\right)$ will be smaller, because the impact on the risk estimates will be somehow proportional before and after intervention, so they largely cancel out. Our results suggest that the precise definition of the stochastic CPM is less relevant for the relative risk estimate after intervention than the question whether variability is included in the CPM.

Also, future research may identify "rules of thumb" that indicate how much the relative risks are overestimated if a simplified CPM is used. "Simpler but not too simple" CPMs may be defined that catch the essential impact of the CPM, without making them unnecessarily complex.

\section{Conclusion}

In this study, we compared the performance of some published stochastic CPMs with a highly simplified CPM, the surrogate "a-factor model", which ignores the variability in the effect of food handling, storage and heat treatment by consumers. The main results of this work showed that the "a-factor model" overestimated the effect of intervention measures in a QMRA, especially if these interventions affect the concentrations. Therefore, the usage of such models may be insufficient for QMRA. We concluded that for an appropriate risk assessment, it may be necessary to include the variation in consumer practices (e.g. variation in storage time and temperature, cooking time and 
temperature and cross-contamination), as described in more realistic and more complex CPMs.

\section{Acknowledgement}

The authors thank Dr. Ana Sofia Ribeiro Duarte for helpful comments on the manuscript.

\section{References}

Bergsma, N. I, Fischer A. H, Van Asselt, E.D., Zwietering, M. H., De Jong, A. E. I. 2007. Consumer food preparation and its implication for survival of Campylobacter jejuni on chicken. British Food Journal 109 (7) 548 - 561.

Berjia, F.L., Andersen, R., Hoekstra, J., Poulsen, M. Nauta, M. (2012.) Risk-benefit assessment of cold-smoked salmon: Microbial risk vs. nutritional benefit. European Journal of Food Research and Review 2(2) 49-68.

Black, R., Levine, M.M., Clements, M.L., Hughes, T.P., Blaser, M.J. 1988. Experimental Campylobacter jejuni infection in humans. Journal of Infectious Diseases 157 472-479.

Bollaerts, K. E., Messens, W., Delhalle, L., Aerts, M., Van Der Stede, Y., Dewulf, J., Quoilin, S., et al. 2009. Development of a quantitative microbial risk assessment for human salmonellosis through household consumption of fresh minced pork meat in Belgium. Risk Analysis 29(6) 820-840.

Bollerslev, A. M., Nauta, M., Hansen, T. B., Aabo, S. 2017. A risk modelling approach for setting microbiological limits using enterococci as indicator for growth potential of Salmonella in pork. International Journal of Food Microbiology 240 102-107. DOI: 10.1016/j.ijfoodmicro.2016.05.007.

Buchanan, R. ., Whiting, R. ., Damert, W. 1997. When is simple good enough: a comparison of the Gompertz, Baranyi, and three-phase linear models for fitting bacterial growth curves. Food Microbiology 14(4) 313-326.

DOI:10.1006/fmic.1997.0125.

Christensen, B., Sommer, H., Rosenquist, H., Nielsen, N. 2001. Risk Assessment on Campylobacter jejuni in Chicken Products. The Danish Veterinary and Food Administration, Denmark.

De Jong, A. E. I., Verhoeff-Bakkenes, L., Nauta, M. J., De Jonge, R. 2008. Crosscontamination in the kitchen: Effect of hygiene measures. Journal of Applied Microbiology 105(2) 615-624. 
Delignette-Muller, M.L., Cornu, M., Pouillot, R., Denis, J.B. 2006. Use of Bayesian modelling in risk assessment: application to growth of Listeria monocytogenes and food flora in cold-smoked salmon. International Journal of Food Microbiology 106 195208.

Duarte, A. S. R., Nauta, M. J., Aabo, S. 2016. Variation in the effect of carcass decontamination impacts the risk for consumers. Food Control 59 12-19.

EFSA (European Food Safety Authority) 2010. Analysis of the baseline survey on the prevalence of Campylobacter in broiler batches and of Campylobacter and Salmonella on broiler carcasses in the EU, 2008, Part A: Campylobacter and Salmonella prevalence estimates. EFSA Journal 8(03) 1503 - 1602

FAO/WHO (Food and Agriculture Organization/World Health Organization) 2002. Risk assessments of Salmonella in eggs and broiler chickens. Microbiological risk assessment series No. 2. Rome, Italy.

FAO/WHO (Food and Agriculture Organization / World Health Organization) 2004. Risk assessment of Listeria monocytogenes in ready-to-eat foods. TECHNICAL REPORT. Microbiological risk assessment series 5. Rome, Italy.

FAO/WHO (Food and Agriculture Organization/World Health Organization) 2009. Risk assessment of Campylobacter in broiler chickens. TECHNICAL REPORT. Microbiological risk assessment series 12 . Rome, Italy.

FDA/FSIS 2003. Quantitative Assessment of Relative Risk to Public Health from Foodborne Listeria monocytogenes Among Selected Categories of Ready-to-Eat Foods. Food and Drug Administration, United States Department of Agriculture, Center for Disease Control.

Gellynck, X., Messens, W., Halet, D., Grijspeerdt, K., Hartnett, E., Viaene, J. 2008. Economics of reducing Campylobacter at different levels within the Belgian poultry meat chain. Journal of Food Protection 71(3) 479-485.

Haas, C.N. 2002 Conditional dose-response relationships for microorganisms: development and application. Risk Analysis 22(3) 455-463.

Havelaar, A. H., Mangen, M.-J. J., de Koeijer, A. A., Bogaardt, M.-J., Evers, E. G., JacobsReitsma, W. F., van Pelt, W., et al. 2007. Effectiveness and efficiency of controlling Campylobacter on broiler chicken meat. Risk Analysis 27(4) 831-44.

Havelaar, A. H., Nauta, M.J., Evers, E. G. (2008). Challenges of quantitative microbial risk assessment at EU level. Trends in Food Science \& Technology 19 26-33.

Hill, A., Simons, R., Ramnial, V., Tennant, J., Cheney, T., Snary, E., et al. 2011.

Quantitative Microbiological Risk Assessment on Salmonella in Slaughter and Breeder 
pigs: Final Report EFSA Supporting Publication 2010; 7(4):EN-46, 463 pp. doi:10.2903/sp.efsa.2010.EN-46

ILSI (International Life Sciences Institute) 2010. Impact of Microbial Distributions on Food Safety. ILSI Europe Report Series. http://ilsi.org/publication/impact-of-microbialdistributions-on-food-safety/

Jørgensen, L.V., Huss, H.H. 1998. Prevalence and growth of Listeria monocytogenes in naturally contaminated seafood. International Journal of Food Microbiology 42 127131.

Nauta, M., Christensen, B. 2011. The Impact of Consumer Phase Models in Microbial Risk Analysis, Risk Analysis 31(2) 255-265.

Nauta, M. J., Fischer, A. R. H., van Asselt, E. D., de Jong, A. E. I., Frewer, L. J., De Jonge, R. 2008. Food safety in the domestic environment: the effect of consumer risk information on human disease risks. Risk Analysis 28(1) 179-92.

Nauta, M. J., Havelaar, A. H. 2008. Risk-based standards for Campylobacter in the broiler meat chain. Food Control 19(4) 372-381.

Nauta, M., Hill, A., Rosenquist, H., Brynestad, S., Fetsch, A., van der Logt, P., Fazil, A., et al. 2009. A comparison of risk assessments on Campylobacter in broiler meat. International Journal of Food Microbiology 129(2) 107-123.

Nauta, M., Kirk, J., Tuominen, P., Ranta, J., Lindqvist, R. 2015. Risk-based microbiological criteria for Campylobacter in broiler meat: A comparison of two approaches. Food Control 53 177-184.

Nauta, M. J., Sanaa, M., Havelaar, A. H. 2012. Risk based microbiological criteria for Campylobacter in broiler meat in the European Union. International Journal of Food Microbiology 158(3) 209-217.

Pouillot, R., Lubran, M. B. 2011. Predictive microbiology models vs. modeling microbial growth within Listeria monocytogenes risk assessment: What parameters matter and why. Food Microbiology 28(4) 720-726.

Ratkowsky, D. A., Olley, J., McMeekin, T. A., Ball, A. 1982. Relationship between temperature and growth rate of bacterial cultures. Journal of Bacteriology 149(1) 1-5.

Redmond, E. C., Griffith, C. J. 2003. Consumer Food Handling in the Home : A Review of Food Safety Studies. Journal of Food Protection 66(1) 130-161.

Ross, T., Rasmussen, S., Fazil, A., Paoli, G., and Sumner, J. (2009). Quantitative risk assessment of Listeria monocytogenes in ready-to-eat meats in Australia. International Journal of Food Microbiology 131(2-3) 128-137. 
Snary, E. L., Swart, A. N., Simons, R. R. L., Coutinho Calado Domingues, A. R., Vigre, H., Evers, E. G., Hill, A. A. 2016. A Quantitative Microbiological Risk Assessment for Salmonella in Pigs for the European Union. Risk Analysis 36(3) 437-449.

Swart, A. N., van Leusden, F., Nauta, M. J. 2016. A QMRA Model for Salmonella in Pork Products During Preparation and Consumption. Risk analysis 36(3) 516-30.

Van Asselt, E. D., De Jong, A. E. I., De Jonge, R., Nauta, M. J. 2008. Cross-contamination in the kitchen: Estimation of transfer rates for cutting boards, hands and knives. Journal of Applied Microbiology 105(5) 1392-1401.

Zwietering, M. H. 2009. Quantitative risk assessment : Is more complex always better? Simple is not stupid and complex is not always more correct. International Journal of Food Microbiology 134(1-2) 57-62. 


\section{Figure captions}

Figure 1 Schematic representation of the Salmonella CPM, adapted from Swart et al. 2016, as explained in 2.3.2. Equivalent parameter names used by Swart et al. (2016) are given in italics, between brackets.

Figure 2 Relative risk estimates obtained after 1,000,000 model iterations for the five case studies: Campylobacter in broiler meat (Campy), Salmonella in pork cuts (Salm PC) and minced pork patties (Salm MM) and Listeria in the healthy population (List HP) and the sensitive population (List SP). Results are given for the stochastic models and the simplified models using the "a-factor", for interventions reducing the mean concentration at retail with $0.5 \log$ and $1 \mathrm{log}$, and reducing and increasing the standard deviation with 0.5 .

Figure 3 The effect of the CPMs in terms of the change in log concentration between retail ( $\left.C_{\text {ret }}, \mathrm{cfu} / \mathrm{g}\right)$ and exposure (dose $\mathrm{D}, \mathrm{cfu} /$ serving). The simplified surrogate models are characterised by the a-factors, given by the black dots. For the stochastic models the change in concentration is variable, given by the cumulative distributions for the four CPMs used. 


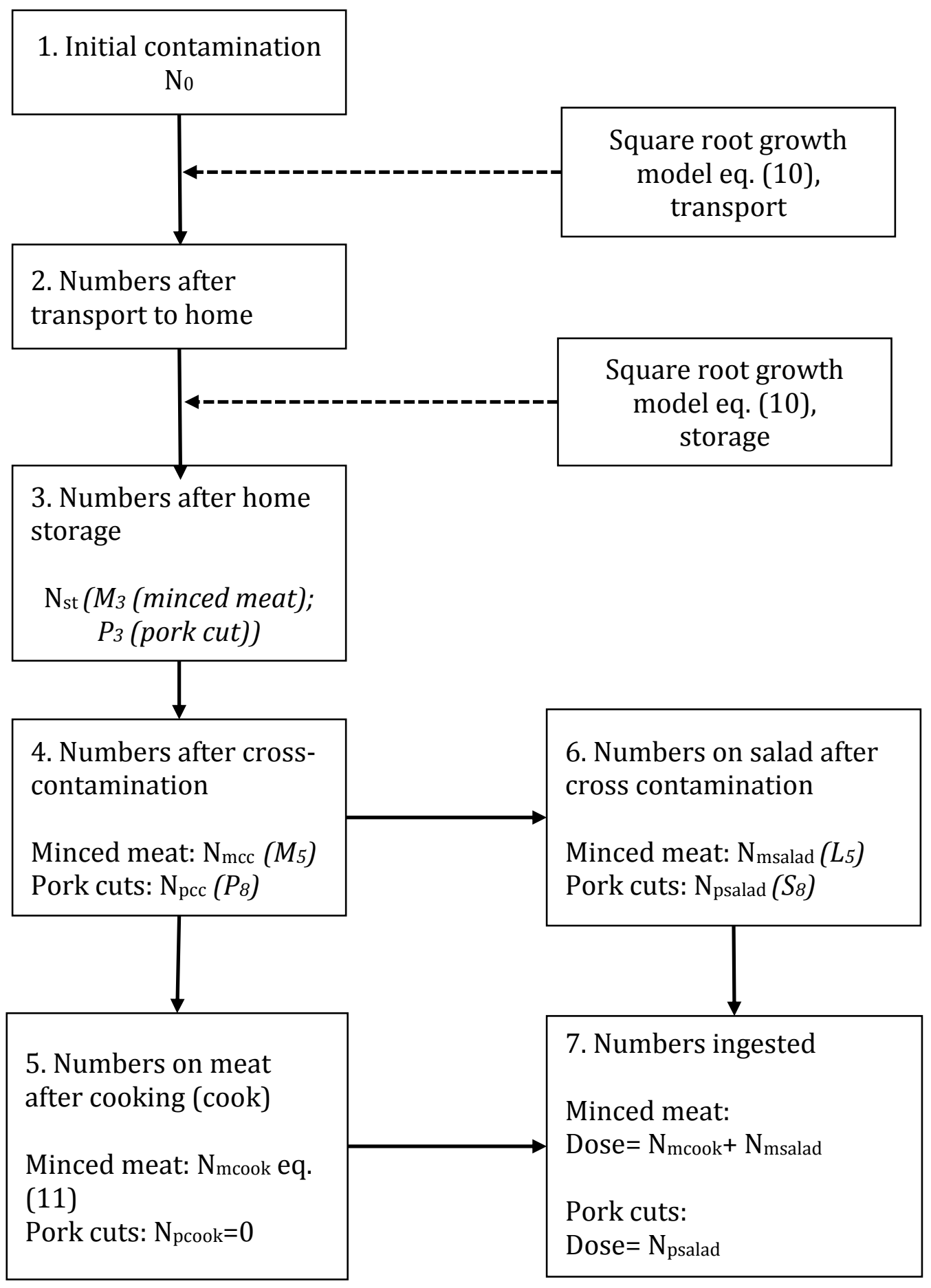

Figure 1 


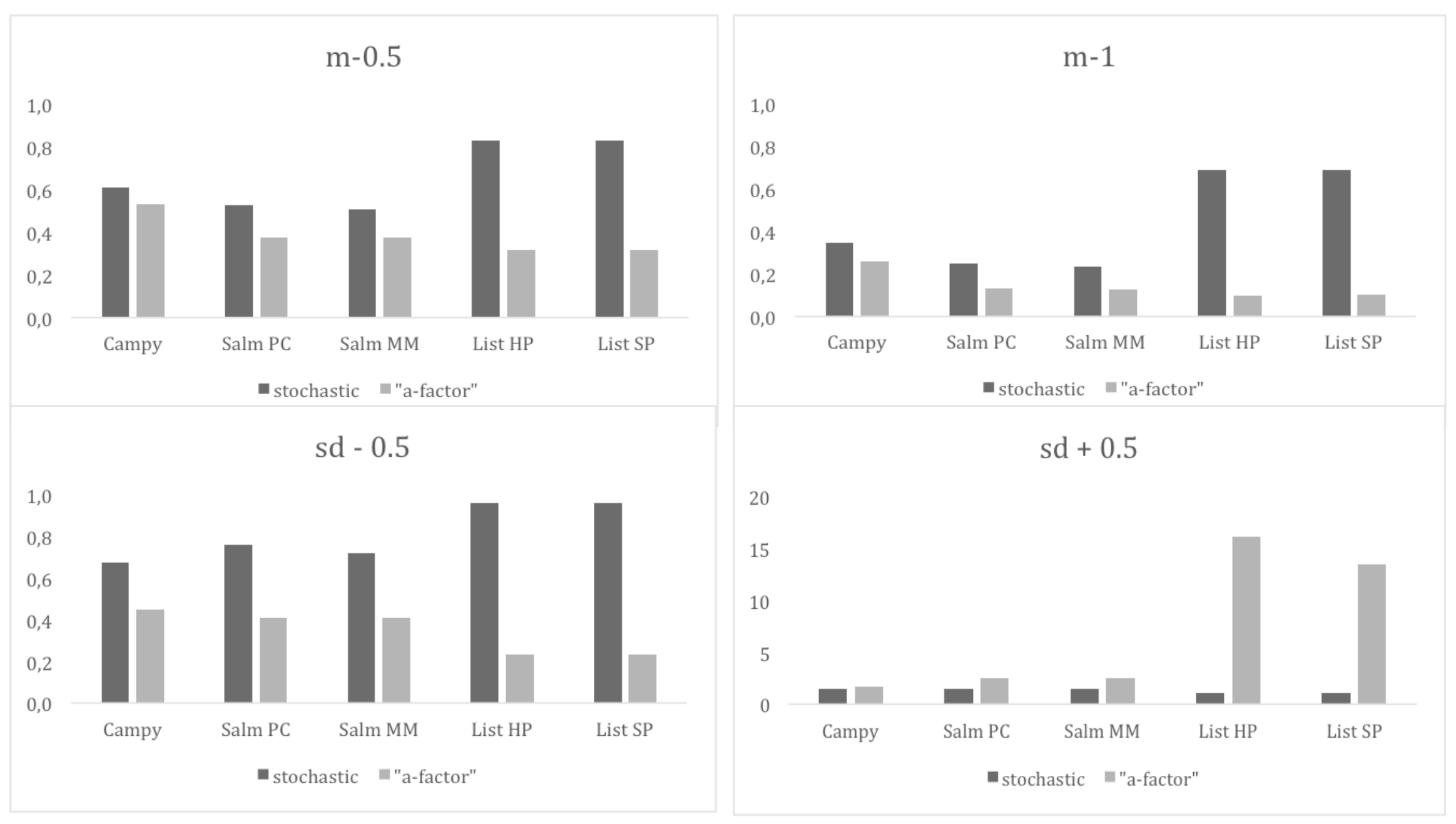

Figure 2 
Neves et al., sept. 2017 ACCEPTED by MICROBIAL RISK ANALYSIS

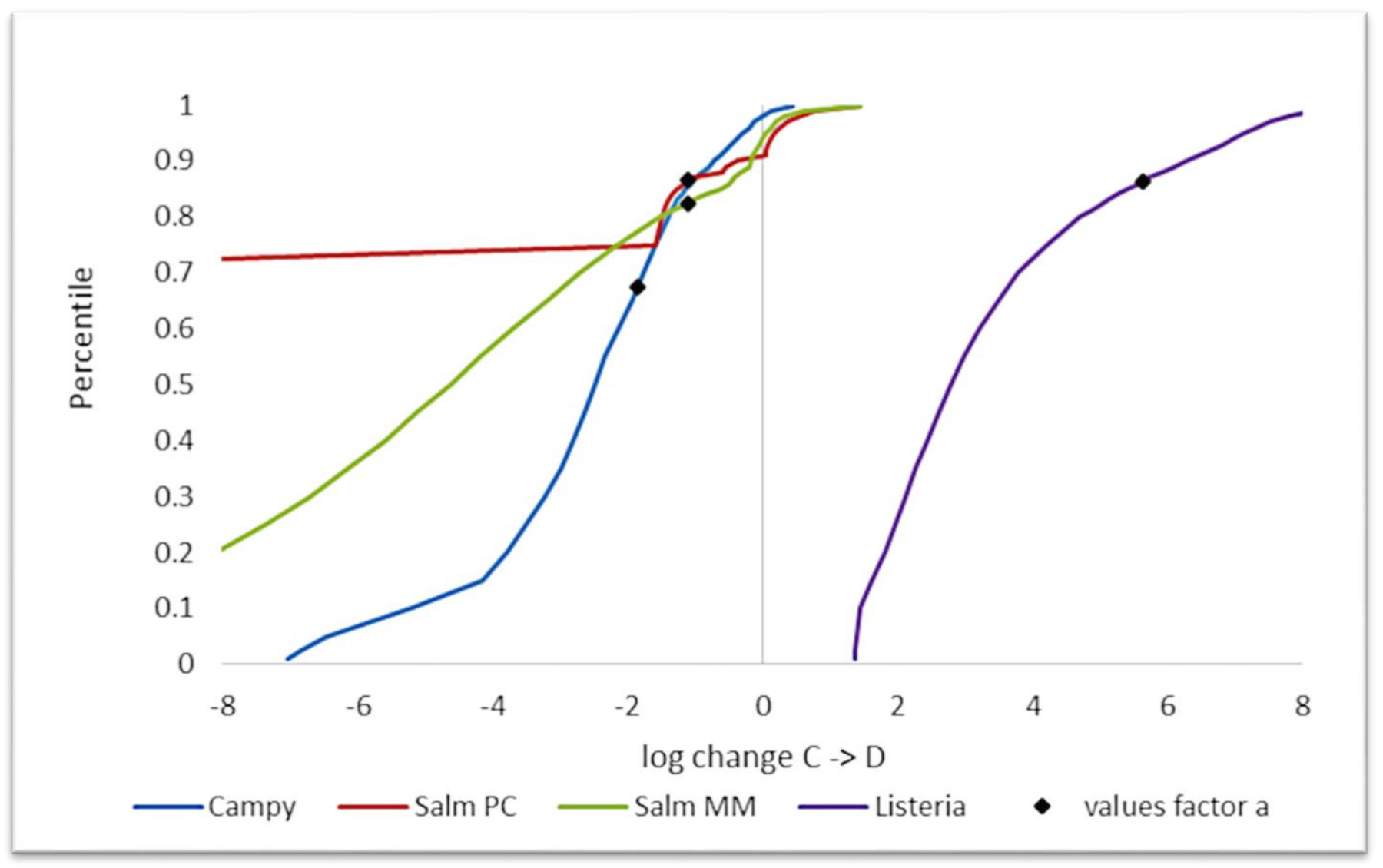

23

$24 \quad$ Figure 3 
Neves et al., sept. 2017 ACCEPTED by MICROBIAL RISK ANALYSIS

26 Table 1 Overview of the case study models, the mean Risk estimate Riskc obtained from eq. (3), and the estimated value for the a-factor.

\begin{tabular}{|c|c|c|c|c|c|}
\hline Case study & Campylobacter & $\begin{array}{l}\text { Salmonella } \\
\text { pork cut }\end{array}$ & Salmonella minced meat & $\begin{array}{l}\text { Listeria } \\
\text { healthy population }\end{array}$ & $\begin{array}{l}\text { Listeria } \\
\text { sensitive population }\end{array}$ \\
\hline Prevalence & 0.25 & 0.12 & 0.12 & 38.5 & 38.5 \\
\hline $\begin{array}{l}\text { Inital concentration } \\
C_{0}(\log \mathrm{cfu} / \mathrm{g})\end{array}$ & Normal $(1.5,1.2)$ & Normal $(1.4,0.7)$ & Normal $(1.4,0.7)$ & Normal $(1.2189,0.8)$ & Normal $(1.2189,0.8)$ \\
\hline Portion size W (g) & $\begin{array}{l}\text { Lognormal (189, } \\
\text { 127) } \max =1000 .\end{array}$ & 146 & 125 & 23 & 23 \\
\hline $\begin{array}{l}\text { Consumer phase } \\
\text { process used for } \\
\text { CPM }\end{array}$ & $\begin{array}{l}\text { Inactivation and } \\
\text { transfer from } \\
\text { meat to salad }\end{array}$ & $\begin{array}{l}\text { Growth and cross } \\
\text { contamination }\end{array}$ & $\begin{array}{l}\text { Growth, } \\
\text { inactivation and transfer } \\
\text { from meat to salad }\end{array}$ & Growth & Growth \\
\hline $\begin{array}{l}\text { Dose response } \\
\text { model } Q_{\text {ill }}\end{array}$ & $\begin{array}{l}\text { BetaPoisson, } \\
\alpha=0.145 \\
\beta=7.59\end{array}$ & $\begin{array}{l}\text { BetaPoisson, } \\
\alpha=0.1324 \\
\beta=51.45\end{array}$ & $\begin{array}{l}\text { BetaPoisson, } \\
\alpha=0.1324 \\
\beta=51.45\end{array}$ & $\begin{array}{l}\text { Exponential } \\
r=2.37 \times 10^{-14}\end{array}$ & $\begin{array}{l}\text { Exponential } \\
r=1.06 \times 10^{-12}\end{array}$ \\
\hline Mean Risk Riskc & 0.0038 & 0.00161 & 0.00156 & $3.43 \times 10^{-7}$ & $1.54 \times 10^{-5}$ \\
\hline a-factor & 0.01427 & 0.0798 & 0.0770 & 425340 & 425910 \\
\hline
\end{tabular}

